\title{
ARTICLE Polydatin attenuates renal fibrosis in diabetic mice through regulating the Cx32-Nox4 signaling pathway
}

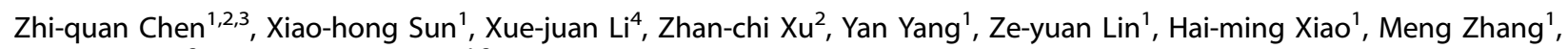 \\ Shi-jian Quan ${ }^{2}$ and He-qing Huang ${ }^{1,2}$
}

We previously found that polydatin could attenuate renal oxidative stress in diabetic mice and improve renal fibrosis. Recent evidence shows that NADPH oxidase 4 (Nox4)-derived reactive oxygen species (ROS) contribute to inflammatory and fibrotic processes in diabetic kidneys. In this study we investigated whether polydatin attenuated renal fibrosis by regulating Nox4 in vitro and in vivo. In high glucose-treated rat glomerular mesangial cells, polydatin significantly decreased the protein levels of Nox4 by promoting its K48-linked polyubiquitination, thus inhibited the production of ROS, and eventually decreasing the expression of fibronectin (FN) and intercellular adhesion molecule-1 (ICAM-1), the main factors that exacerbate diabetic renal fibrosis. Overexpression of Nox4 abolished the inhibitory effects of polydatin on FN and ICAM- 1 expression. In addition, the expression of Connexin32 (Cx32) was significantly decreased, which was restored by polydatin treatment. Cx32 interacted with Nox4 and reduced its protein levels. Knockdown of Cx32 abolished the inhibitory effects of polydatin on the expression of FN and ICAM-1. In the kidneys of streptozocin-induced diabetic mice, administration of polydatin ( $100 \mathrm{mg} \cdot \mathrm{kg}^{-1} \cdot \mathrm{d}^{-1}$, ig, 6 days a week for 12 weeks) increased Cx32 expression and reduced Nox4 expression, decreased renal oxidative stress levels and the expression of fibrotic factors, eventually attenuating renal injury and fibrosis. In conclusion, polydatin promotes K48-linked polyubiquitination and degradation of Nox4 by restoring Cx32 expression, thereby decreasing renal oxidative stress levels and ultimately ameliorating the pathological progress of diabetic renal fibrosis. Thus, polydatin reduces renal oxidative stress levels and attenuates diabetic renal fibrosis through regulating the Cx32-Nox4 signaling pathway.

Keywords: polydatin; diabetic nephropathy; renal fibrosis; Nox4; Cx32; oxidative stress

Acta Pharmacologica Sinica (2020) 41:1587-1596; https://doi.org/10.1038/s41401-020-0475-6

\section{INTRODUCTION}

Diabetic nephropathy (DN) is one of the most serious microvascular complications of diabetes [1]. Currently, there is still a lack of clinically effective drugs for DN. Therapeutic strategies for DN mainly consist of antihypertensive and antihyperlipidemic measures [2]. Therefore, it is extremely important to deeply investigate the pathogenesis of DN and its effective therapeutic drugs.

It is believed that hyperglycemia causes excessive production of reactive oxygen species (ROS) by damaging the mitochondrial electron transport chain, resulting in oxidative stress, which is one of the main causes of DN $[3,4]$. Oxidative stress promotes the formation of advanced glycation end products (AGEs) and the production of proinflammatory and fibrotic factors, resulting in mesangial cell proliferation and accumulation of mesangial matrix [3].

NADPH oxidase 4 (Nox4), a constitutively active Nox, plays an important role in DN [5] and is believed to be the main source of ROS in diabetic kidneys [6]. High glucose (HG)-induced ROS overproduction through upregulation of Nox4 contributes to inflammatory and fibrotic processes in the kidneys $[7,8]$. Studies suggest that the protein level of Nox4 is relevant to K48-linked ubiquitination and subsequent degradation $[9,10]$.

Connexin32 (Cx32), a gap junction protein, is widely expressed in the liver, the kidney, and myocardial tissue [11] and exerts many pharmacological activities; for example, it regulates cell proliferation, apoptosis, transcription, tumors, and liver injury $[12,13]$. Recently, studies have shown that $\mathrm{C} \times 32$ has an inhibitory effect on oxidative stress during liver injury $[13,14]$. Cx32 is downregulated in the diabetic perineurium and chronic liver diseases $[14,15]$. In addition, Cx32 reduces the expression and secretion of renin [16], which regulates the expression of TGF- $\beta 1$ in glomerular messangial cells (GMCs), thereby aggravating DN lesions [17]. Furthermore, we recently demonstrated that $\mathrm{C} \times 32$ ameliorates renal fibrosis in diabetic mice by promoting K48-linked Nox4 polyubiquitination and degradation [18].

Polydatin (PD), the glycoside form of resveratrol, is an active ingredient isolated from Polygonum cuspidatum Sieb. et. Zucc [19]. Polydatin exerts many pharmacological activities; for example, it inhibits inflammation, oxidative stress, and apoptosis, and improves glucose and lipid metabolism [20, 21]. Moreover, our

\footnotetext{
'Laboratory of Pharmacology \& Toxicology, School of Pharmaceutical Sciences, Sun Yat-sen University, Guangzhou 510006, China; ${ }^{2}$ School of Pharmaceutical Sciences,

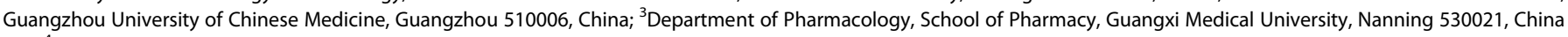
and ${ }^{4}$ Department of Pharmacy, Shenzhen Children's Hospital, Shenzhen 518026, China

Correspondence: Shi-jian Quan (quansj@gzucm.edu.cn) or He-qing Huang (huangheq@mail.sysu.edu.cn)

These authors contributed equally: Zhi-quan Chen, Xiao-hong Sun, Xue-juan Li
}

Received: 21 February 2020 Accepted: 6 July 2020

Published online: 28 July 2020 
previous studies showed that polydatin can inhibit renal oxidative stress and improve diabetic renal fibrosis $[22,23]$.

Nevertheless, the role of polydatin in diabetic renal fibrosis and the specific molecular mechanism of its regulatory effects on oxidative stress remain to be elucidated. In the present study, we aimed to investigate whether polydatin exerts effects against oxidative stress and protects against kidney injury by targeting the Cx32-Nox4 signaling pathway.

\section{MATERIALS AND METHODS}

\section{Reagents and antibodies}

Polydatin for use in animal experiments was obtained from Zelang (purity $>98.0 \%$, HPLC; Jiangsu, China), and polydatin for use in cell experiments was obtained from Chuangwei (Beijing, China). Penicillin, streptomycin, and STZ were purchased from SigmaAldrich Corporation (Missouri, USA). Dulbecco's modified Eagle's medium (DMEM), fetal bovine serum (FBS), MitoSOX ${ }^{\mathrm{TM}}$ Red, RNAiMAX, and Lipofectamine ${ }^{\circledast}$ LTX and Plus reagents were acquired from Life Technologies (New York, USA). MG132 was purchased from Selleck Chemicals (Texas, USA). The total Superoxide Dismutase (SOD) Assay Kit with WST-8 and Lipid Peroxidation MDA Assay Kit were purchased from Beyotime (Jiangsu, China). Enhanced chemiluminescence substrate for the detection of horseradish peroxidase (HRP) was obtained from Thermo Fisher Scientific, Inc. (Illinois, USA). The following plasmids were obtained from Addgene (Massachusetts, USA): mEmerald-Cx32-7, mEmerald-N1, pcDNA3.1-hNox4, pcDNA3.1, and pRK5-HA-Ub-K48.

Antibodies against FN (catalog no. 15613-1-AP), ICAM-1 (catalog no.10020-1-AP), PAl-1 (catalog no. P13801-1-AP) and Nox4 (catalog no. 14347-1-AP) were purchased from Proteintech Group (Illinois, USA); an a-tubulin antibody (catalog no. T8203) was purchased from Sigma-Aldrich Corporation; Cx32 (catalog no. ab66613), Col-IV (catalog no. ab6586) and CTGF (catalog no. ab6992) antibodies were obtained from Abcam (Massachusetts, USA); a Cx32 antibody (catalog no.NBP2-53381) was purchased from Bio-Techne (Minnesota, USA); TGF- $\beta$ (catalog no. 3711) and ubiquitin (catalog no. P4D1) antibodies were obtained from Cell Signaling Technology (Massachusetts, USA); and horseradish peroxidase-conjugated secondary antibodies were obtained from Promega Corporation (Wisconsin, USA). Alexa Fluor ${ }^{\circledR}$ 488conjugated goat anti-rabbit lgG (catalog no. A-11008) and Alexa Fluor $^{\circledR}$ 594-conjugated goat anti-mouse lgG (catalog no. A32742) were purchased from Thermo Fisher Scientific (Illinois, USA).

Primary rat mesangial cell culture

Primary GMCs were isolated from glomeruli cortex fragments from young Sprague-Dawley rats (male, weighing 150-180 g) using a previously described protocol [24].

MTT assay

GMCs were seeded in 96-well plates, and $100 \mu \mathrm{L}$ of DMEM was added to each well. At $70 \%-80 \%$ confluence, the cells were treated with different concentrations of polydatin $(5,10,20,40,80$, or $160 \mu \mathrm{M})$ for $24 \mathrm{~h}$. Then, $10 \mu \mathrm{L}$ of 3-(4,5-dimethylthiazol-2-yl)-2,5diphenyl tetrazolium bromide (MTT, $5 \mathrm{mg} / \mathrm{mL}$, Sigma-Aldrich Corporation) was added to each well to obtain a final concentration of $0.5 \mathrm{mg} / \mathrm{mL}$, and the cells were incubated at $37^{\circ} \mathrm{C}$ for $4 \mathrm{~h}$. The medium was carefully discarded, $200 \mu \mathrm{L}$ of DMSO was added to dissolve the formazan crystals, and the absorbance was measured at $490 \mathrm{~nm}$ using a microplate reader (Bio-Tek, Vermont, USA).

Transfection of plasmids, short hairpin RNAs and small interfering RNAs

Plasmids and RNAs were transfected into GMCs as previously described [18]. Three short hairpin RNAs (shRNAs) targeting Nox4 and a small interfering RNA (siRNA) targeting Cx32 were synthesized by Genechem (Shanghai, China). The sequences of Nox4 and Cx32 were previously reported [18]. After further treatment, the cells were harvested, and Western blot analysis was performed.

\section{Western blot analysis}

Western blot analysis was performed as previously described [25].

Immunofluorescence staining

Immunofluorescence was performed as previously described [18].

Immunoprecipitation assay

Immunoprecipitation assays were performed as previously described [18].

Detection of intracellular superoxide and $\mathrm{H}_{2} \mathrm{O}_{2}$ levels

The fluorescent probe dihydroethidium (DHE, Beyotime, Jiangsu, China) was used according to the instructions to detect intracellular superoxide levels. Cells were washed twice with PBS and then loaded with DHE $(10 \mu \mathrm{m})$ in fresh DMEM for $30 \mathrm{~min}$ at $37^{\circ} \mathrm{C}$. Mitochondrial superoxide formation was detected by incubating cells in the dark with MitoSOX Red dye $(5 \mu \mathrm{m})$ for $30 \mathrm{~min}$ at $37^{\circ} \mathrm{C}$. The fluorescence was then quantified, and images were collected with the ArrayScan VTI 600 Plus system. In addition, the $\mathrm{H}_{2} \mathrm{O}_{2}$ levels in GMCs were measured using a hydrogen peroxide detection kit according to the instructions.

Measurement of mitochondrial superoxide production Mitochondrial superoxide levels were detected by live-cell imaging using MitoSOX Red as previously described [18].

\section{Animals and treatment}

All animal care and experimental procedures complied with the Guide for the Care and Use of Laboratory Animals (NIH Publication No. 85-23, revised 1996), were in line with the China Animal Welfare Legislation, and were approved by the Ethics Committee on the Care and Use of Laboratory Animals of Sun Yat-sen University, Guangzhou, China. In addition, the protocol was approved by the IACUC (approval no. IACUC-DB-17-2017).

C57BL/6 mice (male, 6-8-week old, weighing $20 \pm 2 \mathrm{~g}$, specific pathogen-free (SPF) grade) were obtained from the Laboratory Animal Centre, Sun Yat-sen University (Guangdong, China). All animals were housed under specific pathogen-free conditions and in a temperature-controlled $\left(20-25{ }^{\circ} \mathrm{C}\right)$ and humidity-controlled (40\%-70\%) barrier system with a 12-h:12-h light and dark cycle.

Diabetes was induced in mice as previously described [22]. Polydatin was dissolved in $0.5 \%$ (w/v) CMC-Na. Diabetic mice were randomly divided into two groups ( $n=10$ in each group) and were administered polydatin $(100 \mathrm{mg} / \mathrm{kg})$ or an equal volume of $0.5 \%(\mathrm{w} / \mathrm{v}) \mathrm{CMC}-\mathrm{Na}$ by gavage. The mice were treated with polydatin daily between 9:30 and 10:30 6 days a week for 12 weeks. Fasting blood glucose (FBG) levels were detected once a week with a one-touch glucometer (Johnson, New Jersey, USA). At the termination of the experiment, the mice were placed in metabolic cages overnight to collect urine. The next day, the body weight of each mouse was recorded, the mice were anesthetized, and blood samples were obtained. Finally, the animals were killed by cervical dislocation, and both kidneys were quickly removed and weighed.

Statistical analysis

Data analysis was performed in a blinded manner using GraphPad Prism 5.0. The values are expressed as the mean \pm SD. Unpaired Student's $t$-test was used to compare two groups. For multiple comparisons, the data were analyzed by one-way ANOVA with post hoc multiple comparisons. $P<0.05$ was considered to be statistically significant. Independent experiments were performed at least three times with similar results. 


\section{RESULTS}

Polydatin inhibits the expression of FN and ICAM-1 by reducing the production of superoxide and $\mathrm{H}_{2} \mathrm{O}_{2}$ in $\mathrm{HG}$-exposed GMCs The deposition of extracellular matrix proteins such as $\mathrm{FN}$ in the glomerular mesangial area and the secretion of ICAM-1 are the main factors that exacerbate diabetic renal fibrosis [26-28].
Therefore, inhibiting the upregulation of FN and ICAM-1 induced by HG in GMCs is of great significance to prevent and treat DN. Western blot analysis was performed to examine the effects of HG treatment for different lengths of time on the expression of FN and ICAM-1. The results showed that treatment with HG (30 mM) for $24 \mathrm{~h}$ significantly increased the expression of FN (Fig. 1a) and a

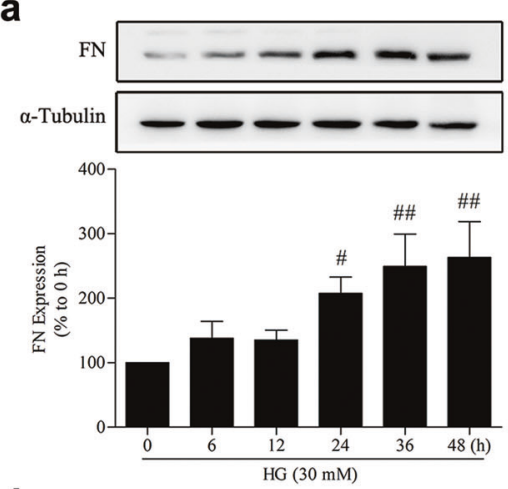

d
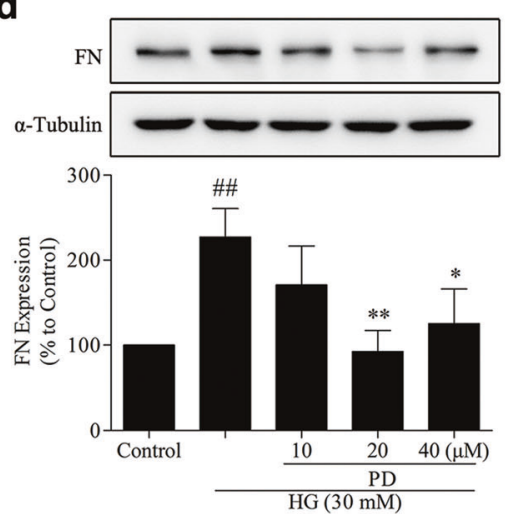

b

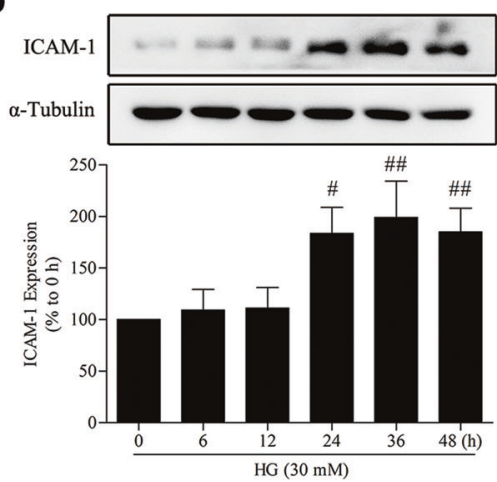

e
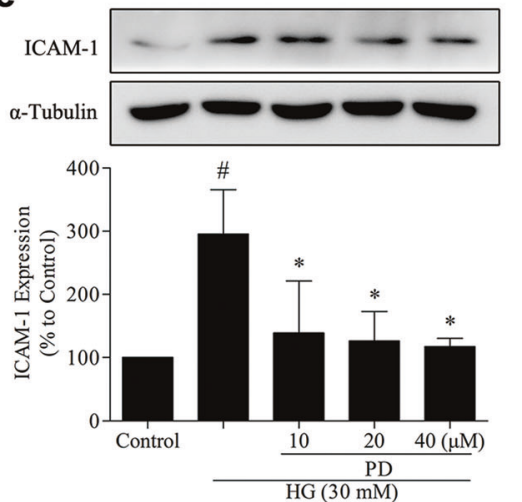

C

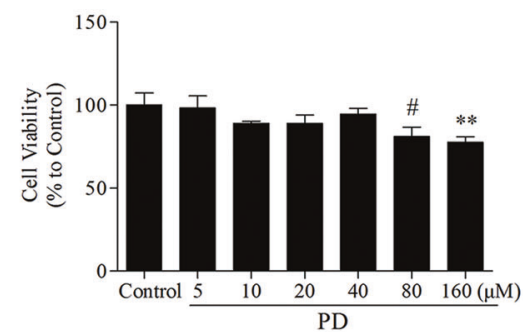

f

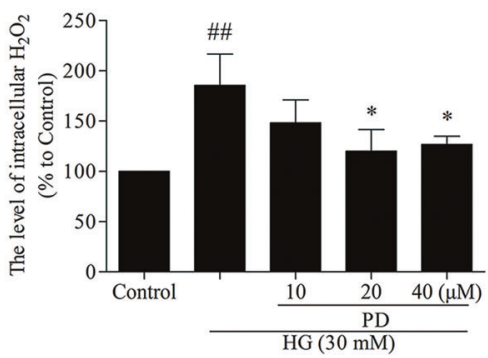

g

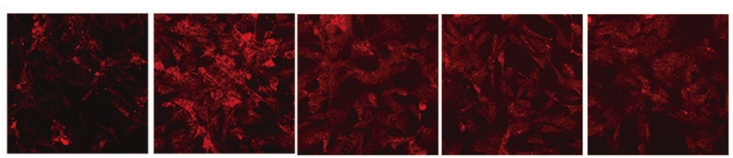

h

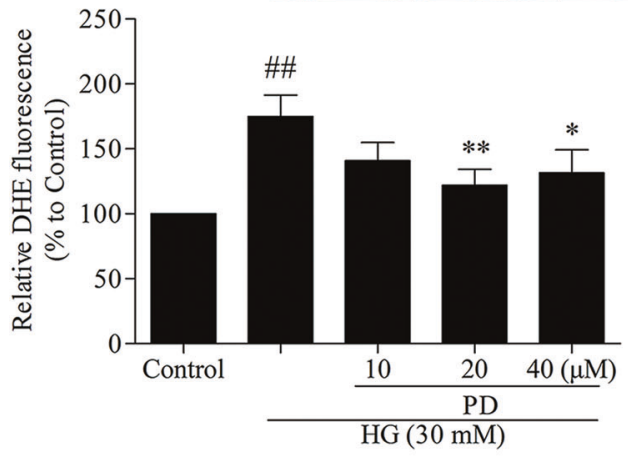

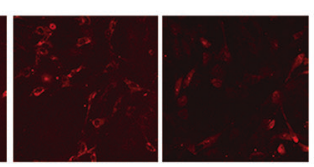
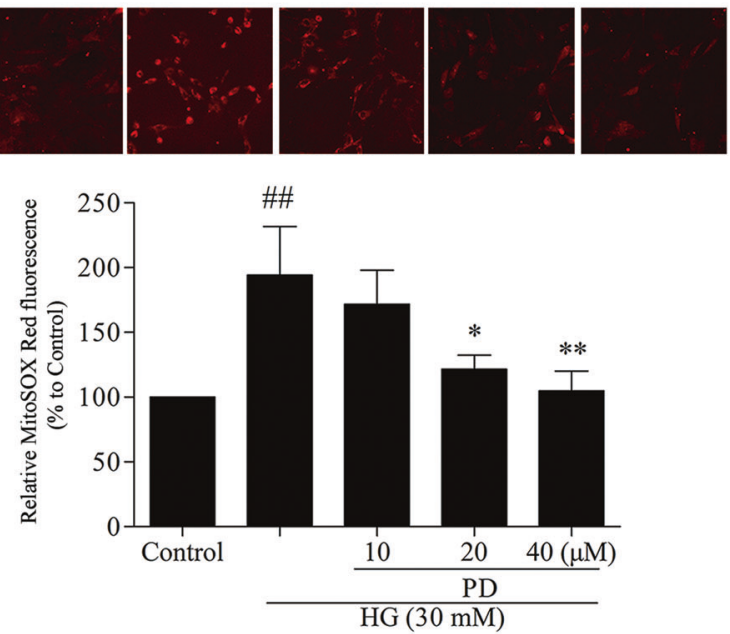

Fig. 1 Polydatin inhibits the expression of FN and ICAM-1 by reducing the production of superoxide and $\mathrm{H}_{2} \mathrm{O}_{2}$ in $\mathrm{HG}-$ exposed GMCs. $\mathbf{a}$, $\mathbf{b}$ Western blot analysis was performed to examine the effects of $\mathrm{HG}(30 \mathrm{mM})$ exposed for different amounts of time on the expression of FN and ICAM-1; ${ }^{\#} P<0.05,{ }^{\#} P<0.01$ vs. 0 h. c GMCs cultured in normal glucose (NG, $\left.5.5 \mathrm{mM}\right)$ were treated with different concentrations of polydatin for $24 \mathrm{~h}$. Polydatin had no toxic effects on GMCs when the concentration was less than $80 \mu \mathrm{M}$, as revealed by the MTT assay; ${ }^{\#} P<$ $0.05,{ }^{\# \#} P<0.01$ vs. control. d, e After pretreatment with different concentrations of polydatin $(10,20$, or $40 \mu \mathrm{M})$ for $2 \mathrm{~h}$, GMCs were exposed to $\mathrm{HG}(30 \mathrm{mM})$ for $24 \mathrm{~h}$ in the presence or absence of polydatin. At concentrations of 20 and $40 \mu \mathrm{M}$, polydatin decreased the expression of FN and ICAM-1 in HG-exposed GMCs; ${ }^{\#} P<0.05$, \#\# $P<0.01$ vs. control; ${ }^{*} P<0.05,{ }^{* *} P<0.01$ vs. HG. f-h After pretreatment with different concentrations of polydatin $(10,20$ or $40 \mu \mathrm{M})$ for $2 \mathrm{~h}, \mathrm{GMCs}$ were exposed to HG $(30 \mathrm{mM})$ for $12 \mathrm{~h}$ in the presence or absence of polydatin. A hydrogen peroxide detection kit, $\mathrm{DHE}$, and MitoSOX Red were used to detect the levels of hydrogen peroxide, superoxide, and mitochondrial superoxide, respectively, in GMCs; ${ }^{\# \#} P<0.01$ vs. control; ${ }^{*} P<0.05,{ }^{* *} P<0.01$ vs. HG. HG high glucose, PD polydatin, control represents the group exposed to normal glucose $(\mathrm{NG}, 5.5 \mathrm{mM})$. The above experiments were performed at least three times with similar results. 
ICAM-1 (Fig. 1b) in GMCs. Thus, HG (30 mM) treatment for $24 \mathrm{~h}$ was used for subsequent experiments. As shown in Fig. 1c, the MTT assay revealed that polydatin showed no toxic effects on GMCs when the concentration was less than $80 \mu \mathrm{M}$. Therefore, polydatin was used at concentrations of 10,20 , and $40 \mu \mathrm{M}$ for subsequent experiments. At concentrations of 20 and $40 \mu \mathrm{M}$, polydatin significantly reduced the expression of FN (Fig. 1d) and ICAM-1 (Fig. 1e) in HG-exposed GMCs.

A growing body of evidence suggests that polydatin has a significant inhibitory effect on oxidative stress [29-31]. Thus, we used a hydrogen peroxide detection kit, DHE and MitoSOX Red to detect the levels of hydrogen peroxide $\left(\mathrm{H}_{2} \mathrm{O}_{2}\right)$, superoxide and mitochondrial superoxide, respectively, in GMCs. We found that polydatin significantly reduced the excessive production of $\mathrm{H}_{2} \mathrm{O}_{2}$ (Fig. 1f), superoxide (Fig. 1g), and mitochondrial superoxide (Fig. 1h) in HG-exposed GMCs.

Nox4 is involved in the regulation of FN and ICAM-1 expression by polydatin in HG-exposed GMCs

Studies have shown that oxidative stress plays a key role in the pathological progression of DN [32]. Our previous studies and others have shown that polydatin has a significant antioxidant protective effect in DN $[22,33]$. Nevertheless, the exact role of polydatin in diabetic renal fibrosis and the specific molecular mechanism of its regulatory effects on oxidative stress remain to be elucidated. Although there are many sources of ROS in cells and tissues, such as xanthine oxidase (XO) and the synthesis of uncoupled nitric oxide (NOSs), the main sources of ROS in the diabetic kidneys are the mitochondrial electron transport chain and NADPH oxidases, especially Nox4 $[6,8]$. Consistent with previously reported results [34], we found that the expression of Nox4 was obviously increased under HG conditions (Fig. 2a). Western blot analysis was performed to analyze the effects of three Nox4-targeting shRNAs on Nox4 protein levels. The results showed that all three shRNAs significantly decreased the protein levels of Nox4 and that shRNA-4 had the most robust effect (Fig. 2b). Thus, shRNA-4, which targeted Nox4, was used for subsequent experiments. Next, GMCs were transfected with the Nox4 shRNA-4 under HG conditions. Compared with HG exposure, Nox4 depletion reduced the expression of FN and ICAM-1 (Fig. 2c, d) in GMCs. Importantly, polydatin significantly reduced Nox4 protein levels in HGexposed GMCs (Fig. 2e). Furthermore, the downregulation of FN and ICAM-1 expression by polydatin was blocked by Nox4 a

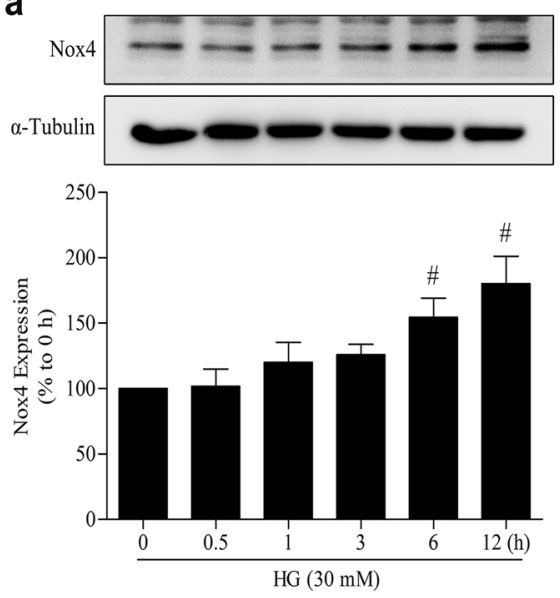

d
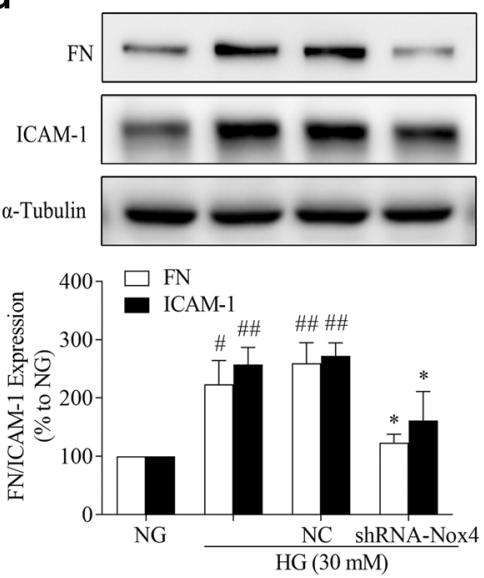

b
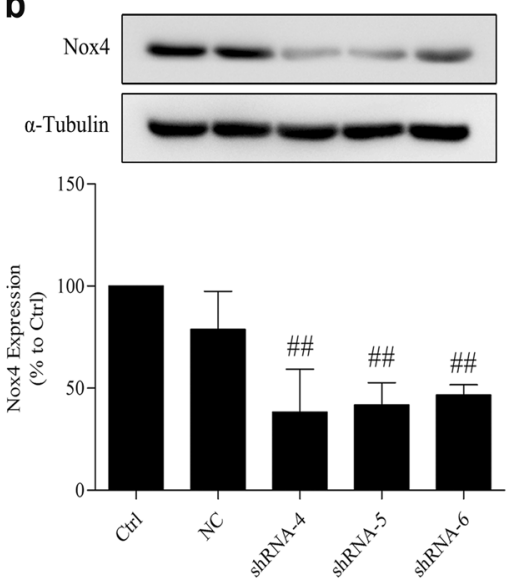

e
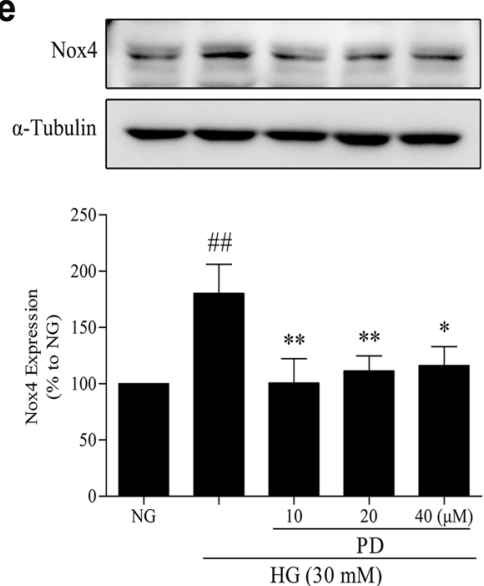

C
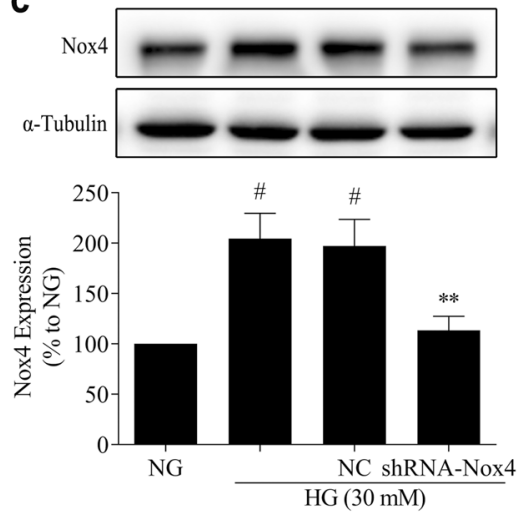

f

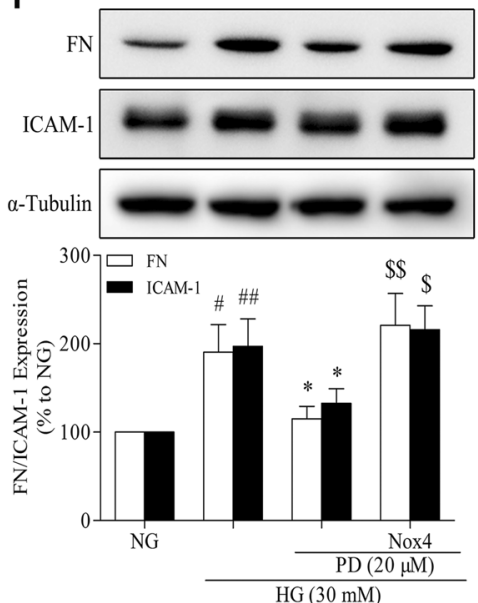

Fig. 2 Nox4 is involved in the regulation of FN and ICAM-1 expression by polydatin in HG-exposed GMCs. a The expression of Nox4 was obviously increased under HG $(30 \mathrm{mM})$ conditions; ${ }^{\#} P<0.05 \mathrm{vs}$. $0 \mathrm{~h}$. b Western blot analysis was performed to analyze the effects of three Nox4-targeting shRNAs on the protein levels of Nox4, ${ }^{\#} P<0.01$ vs. control. c, d Compared with the HG treatment (30 mM, $24 \mathrm{~h}$ ), Nox4 depletion reduced the expression of FN and ICAM-1 in GMCs; ${ }^{*} P<0.05,{ }^{\#} P<0.01$ vs. NG; ${ }^{*} P<0.05$, ${ }^{*} P<0.01$ vs. HG. e Polydatin significantly reduced Nox4 protein levels in GMCs exposed to HG for $12 \mathrm{~h}$; ${ }^{\# \#} P<0.01$ vs. NG; ${ }^{*} P<0.05$, ${ }^{* *} P<0.01$ vs. HG. $f$ The ability of polydatin $(20 \mu \mathrm{M})$ to downregulate FN and ICAM-1 was blocked by Nox4 overexpression in GMCs exposed to HG for $24 \mathrm{~h}$; ${ }^{\#} P<0.05$, \#\# $P<0.01$ vs. NG; ${ }^{*} P<0.05$ vs. HG; ${ }^{\$} P<0.05, \$ \$ P<0.01$ vs. HG + PD. HG high glucose, Ctrl control, NC negative control, NG normal glucose, PD polydatin. The above experiments were performed at least three times with similar results. 

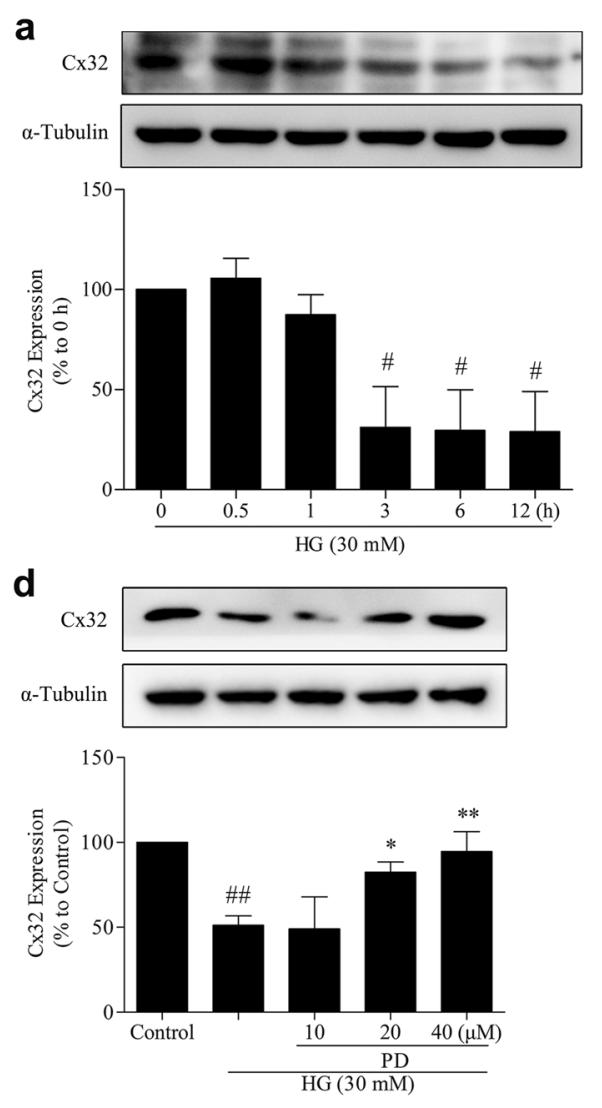

b
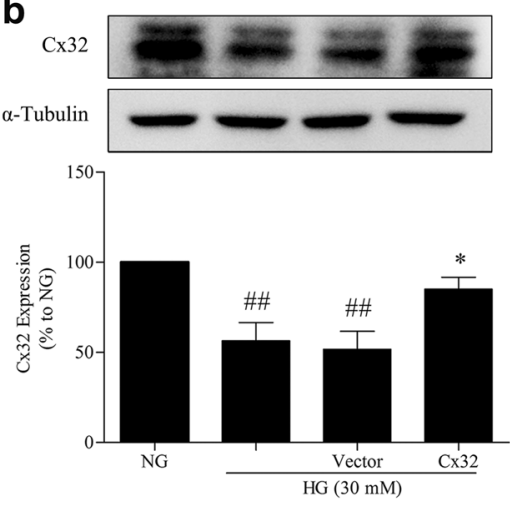

e

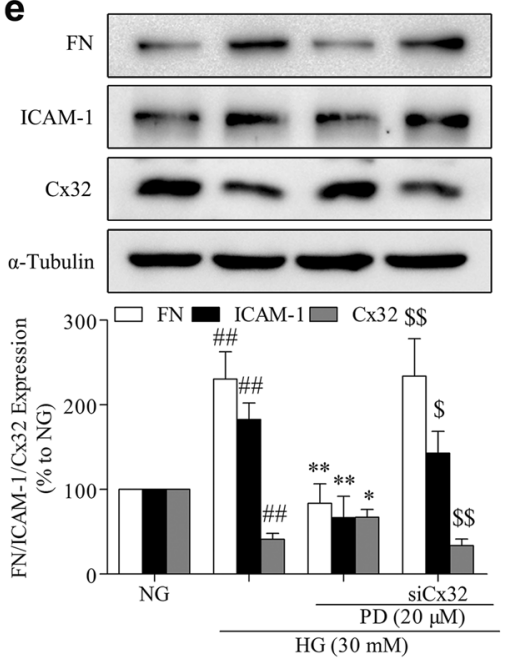

C

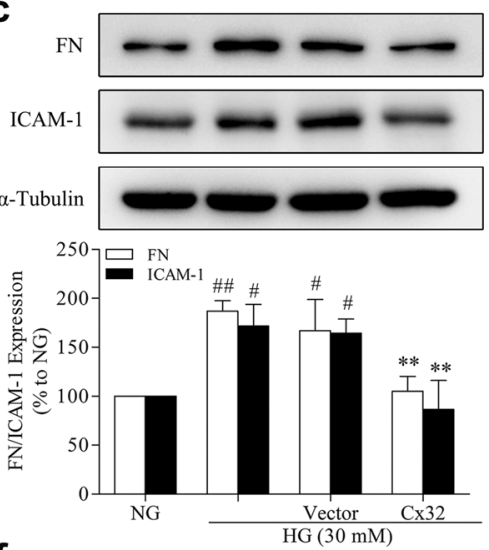

f
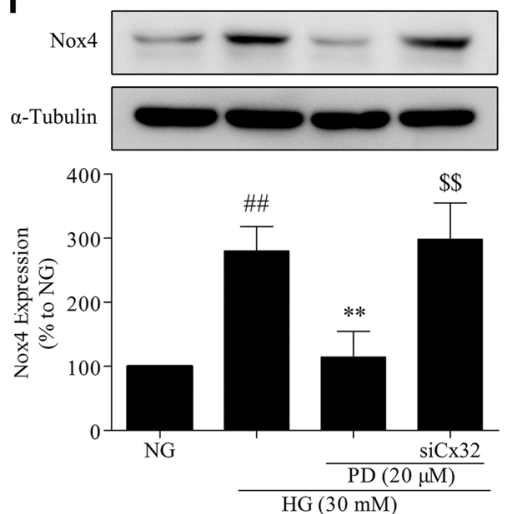

Fig. 3 Cx32 mediates the regulation of Nox4 expression by polydatin in HG-exposed GMCs. a The expression of Cx32 was downregulated in GMCs after exposure to HG $(30 \mathrm{mM})$ for $3 \mathrm{~h}$; ${ }^{\#} P<0.05$ vs. $0 \mathrm{~h}$. b, c We observed that the transfection of the Cx32 plasmid into HG-exposed GMCs reduced the expression of FN and ICAM-1 by restoring Cx32 protein levels; ${ }^{\#} P<0.05,{ }^{\# \#} P<0.01$ vs. NG; ${ }^{*} P<0.05,{ }^{* *} P<0.01$ vs. HG. d After pretreatment with different concentrations of polydatin $(10,20$, or $40 \mu \mathrm{M})$ for $2 \mathrm{~h}$, GMCs were exposed to HG (30 mM) for $3 \mathrm{~h}$ in the presence or absence of polydatin. Polydatin increased the expression of Cx32 in a dose-dependent manner in GMCs exposed to HG; ${ }^{\# \#} P<0.01$ vs. control; ${ }^{*} P<0.05,{ }^{*} P<0.01$ vs. HG. e, f After pretreatment with polydatin $(20 \mu \mathrm{M})$ for $2 \mathrm{~h}, \mathrm{GMCs}$ were treated with $\mathrm{HG}(30 \mathrm{mM})$ for $24 \mathrm{~h}$ in the presence or absence of polydatin. Depletion of $\mathrm{Cx} 32$ abolished the ability of polydatin to downregulate FN and ICAM-1 expression as well as the effect of polydatin on Nox4 in GMCs exposed to HG conditions; ${ }^{\# \#} P<0.01$ vs. NG; ${ }^{*} P<0.05,{ }^{* *} P<0.01$ vs. $H G$; ${ }^{\$} P<0.05$, ${ }^{\$} P<0.01$ vs. HG + PD. HG high glucose, NG normal glucose, PD polydatin. The above experiments were performed at least three times with similar results.

overexpression (Fig. 2f), indicating that polydatin may reduce the overproduction of ROS in GMCs by decreasing the protein levels of Nox4, thereby inhibiting the upregulation of $\mathrm{FN}$ and ICAM-1 expression.

Cx32 mediates the regulation of Nox4 expression by polydatin in HG-exposed GMCs

To determine whether $\mathrm{C} \times 32$ mediates the regulation of Nox4 expression by polydatin, we initially investigated the role of $\mathrm{Cx} 32$ in renal fibrosis. As shown in Fig. 3a, the expression of $\mathrm{Cx} 32$ was downregulated in GMCs by HG treatment for $3 \mathrm{~h}$. We observed that transfection of the Cx32 plasmid into HG-exposed GMCs reduced the expression of FN and ICAM- 1 by restoring $\mathrm{C} \times 32$ protein levels (Fig. 3b, c). Concurrently, we found that polydatin increased the expression of $\mathrm{C} \times 32$ in a dose-dependent manner in HG-exposed GMCs (Fig. 3d). Furthermore, depletion of Cx32 in GMCs under HG conditions abolished the ability of polydatin to downregulate FN and ICAM-1 expression (Fig. 3e) as well as the effect of polydatin on Nox4 (Fig. 3f), suggesting that Cx32 at least partially mediates the regulation of Nox4 expression by polydatinin in HG-exposed GMCs.
Cx32 interacts with Nox4 and inhibits its expression in GMCs To further investigate the relationship between Cx32 and Nox4, we performed immunofluorescence analysis to identify the subcellular distribution of both $\mathrm{C} \times 32$ and Nox4 in GMCs. We observed that Cx32 (red) colocalized with Nox4 (green) in GMCs under normal conditions (Fig. 4a), providing spatial potential for interactions between Cx32 and Nox4. Importantly, the immunoprecipitation results revealed that $\mathrm{C} \times 32$ indeed interacted with Nox4 in GMCs under normal conditions (Fig. 4b). Transfection of the $\mathrm{C} \times 32$ plasmid into GMCs under HG conditions downregulated the expression of Nox4 by restoring Cx32 protein levels (Fig. 4c). Meanwhile, transfection of $\mathrm{C} \times 32$ siRNA into GMCs under HG conditions further promoted Nox4 expression by reducing the protein levels of Cx32 (Fig. 4d).

Polydatin promotes K48-linked polyubiquitination of Nox4 by Cx32 in HG-exposed GMCs

Considering that Nox4 is a continuously activated oxidase, its protein levels are closely related to K48-linked polyubiquitination and subsequent degradation $[9,10,35]$; therefore, we assessed whether polydatin reduces the expression of Nox4 by regulating 

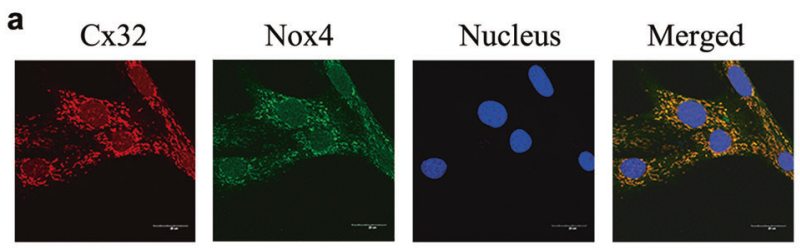

b

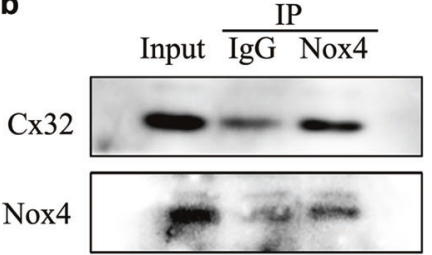

d

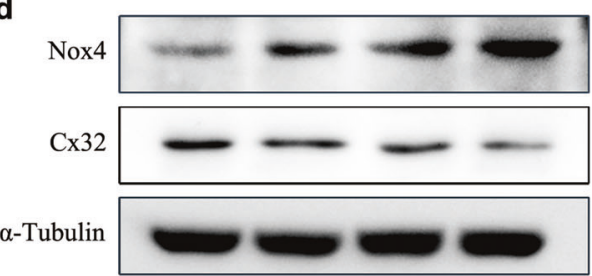

C
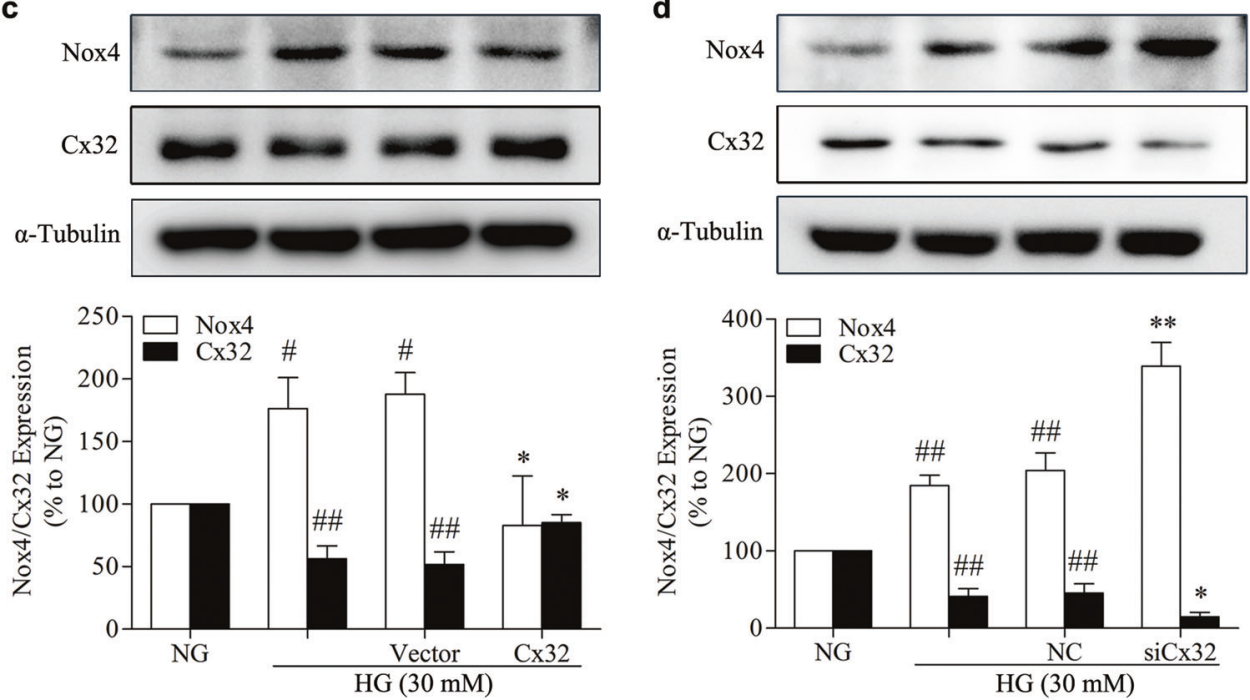

Fig. 4 a Immunofluorescence analysis was performed to identify the subcellular distribution of both Cx32 and Nox4 in GMCs. Red fluorescence indicates the localization of $\mathrm{C} \times 32$. Green fluorescence indicates Nox4. Blue fluorescence indicates nuclei. $\mathbf{b} \mathrm{C} \times 32$ interacted with Nox4 in GMCs. c Transfection of the Cx32 plasmid into GMCs under HG conditions downregulated the expression of Nox4 by restoring Cx32 protein levels; ${ }^{\#} P<0.05,{ }^{\# \#} P<0.01$ vs. NG; ${ }^{*} P<0.05$ vs. HG. d Transfection of $C \times 32$ siRNA into GMCs under HG conditions further promoted Nox4 expression by reducing the protein levels of $C \times 32 ;{ }^{\# \#} P<0.01$ vs. NG; ${ }^{*} P<0.05,{ }^{* *} P<0.01$ vs. HG. IP immunoprecipitation, HG high glucose, NG normal glucose, NC negative control. The above experiments were performed at least three times with similar results.

a
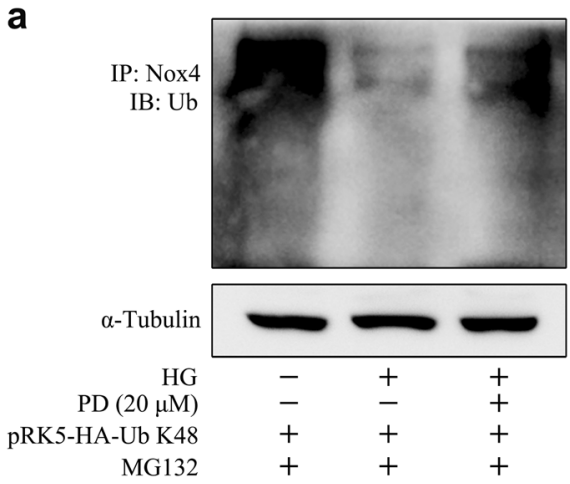

b

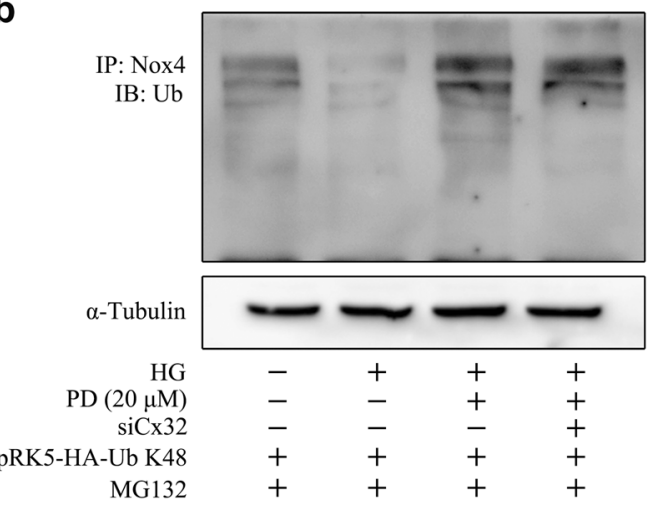

Fig. 5 Polydatin promotes K48-linked polyubiquitination of Nox4 by Cx32 in HG-exposed GMCs. The pRK5-HA-Ub K48 plasmid was transfected into GMCs in the presence or absence of Cx32 siRNA. After $24 \mathrm{~h}$, the cells were pretreated with polydatin ( $20 \mu \mathrm{M})$ for $2 \mathrm{~h}$ and then stimulated with HG for $12 \mathrm{~h}$ in the presence or absence of polydatin $(20 \mu \mathrm{M})$. MG132 $(5 \mu \mathrm{M})$ was added $2 \mathrm{~h}$ before the end of the treatment. a Polydatin treatment promoted the K48-linked polyubiquitination of Nox4 in HG-exposed GMCs. b Depletion of Cx32 under HG conditions abolished the effects of polydatin on the K48-linked polyubiquitination of Nox4. HG high glucose, PD polydatin.

its K48-linked polyubiquitination. We observed that compared with control treatment, HG treatment for $12 \mathrm{~h}$ reduced the K48linked polyubiquitination of Nox4 in GMCs, and this effect was reversed by polydatin treatment (Fig. 5a). Interestingly, we found that depletion of $\mathrm{C} \times 32$ under HG conditions abolished the effects of polydatin on the K48-linked polyubiquitination of Nox4 (Fig. $5 \mathrm{~b}$ ). Combined, these data suggest that polydatin may promote K48linked polyubiquitination of Nox4 by $\mathrm{C} \times 32$, thereby reducing Nox4 protein levels.
Polydatin improves renal dysfunction and fibrosis in diabetic mice by restoring $\mathrm{C} \times 32$ expression and reducing Nox4 expression in the kidneys of diabetic mice

In vitro experiments have confirmed that polydatin can reduce the overproduction of ROS in HG-exposed GMCs by decreasing the expression of Nox4 and restoring the expression of $\mathrm{C} \times 32$, thus retarding the expression of FN and ICAM-1. To validate the findings we observed in vitro, we utilized the STZ-induced diabetic mouse model to investigate the effects of polydatin on 
a

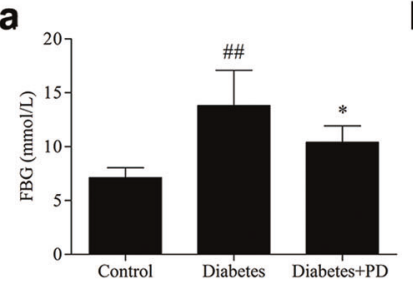

e

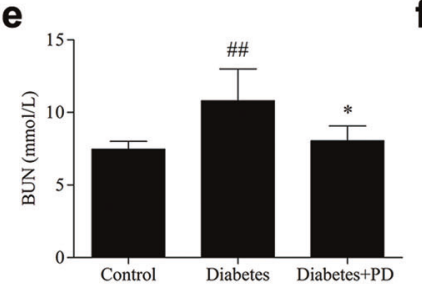

h

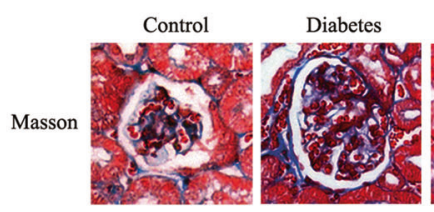

i

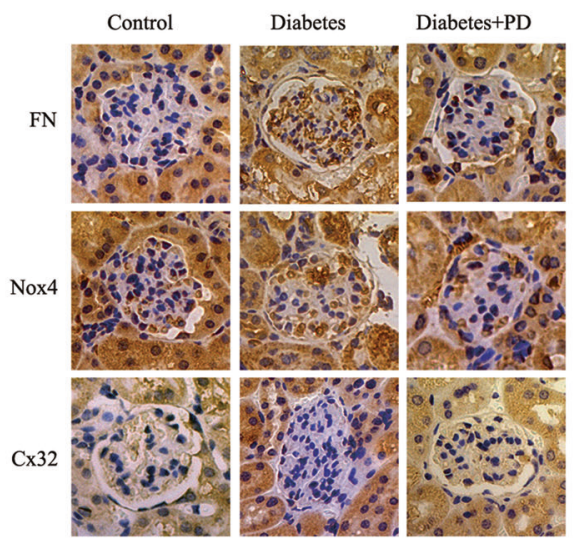

C

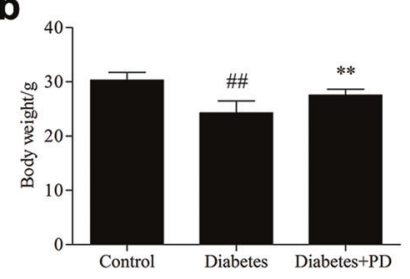

f

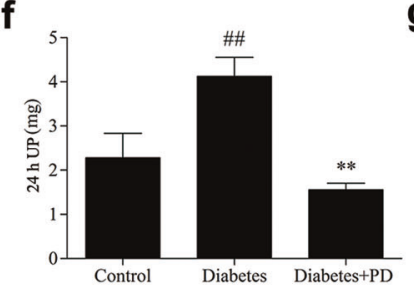

g d

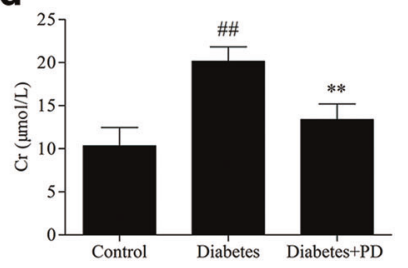

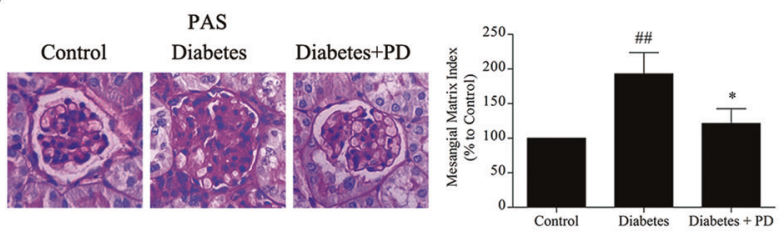
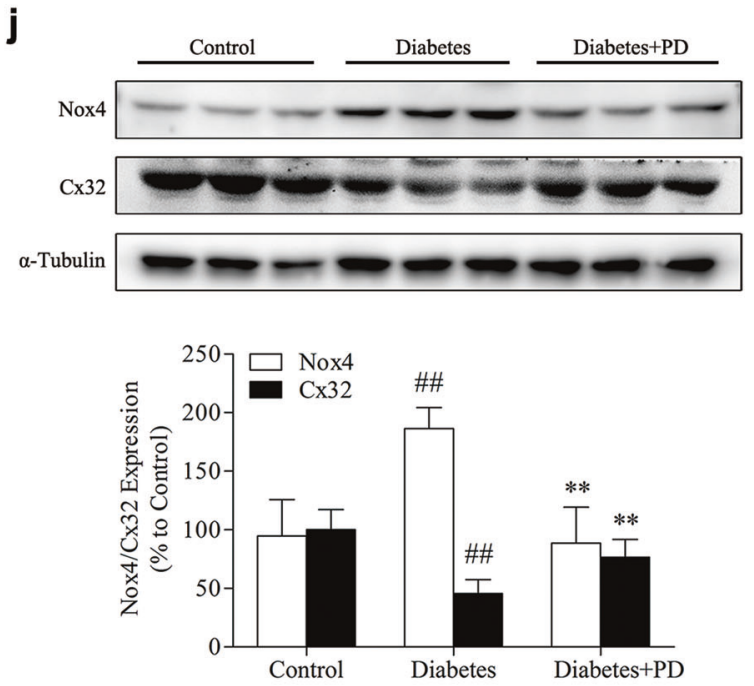

Fig. 6 Polydatin improves renal dysfunction and fibrosis in diabetic mice by restoring Cx32 expression and reducing Nox4 expression in the kidneys of diabetic mice. $\mathbf{a}, \mathbf{b}$ The FBG levels and body weights of diabetic mice were analyzed. $\mathbf{c}$ The KW/BW ratio was calculated to evaluate the kidney hypertrophy index. $\mathbf{d}$-f Serum $\mathrm{Cr}$, serum BUN, and 24-h UP levels were detected to assess renal injury. g, $\mathbf{h}$ Glomerular histopathology analysis of the kidneys of diabetic mice was performed by periodic acid-Schiff (PAS) staining and Masson staining ( $\times 400$ magnification). $\mathbf{i}$ The expression levels of FN, Nox4, and Cx32 in the glomeruli were evaluated by immunohistochemical staining ( $\times 400$ magnification). $\mathbf{j}$ The expression levels of Nox4 and $\mathrm{C} \times 32$ in the kidneys of mice were detected using Western blot analysis. $\# \#<<0.01$ vs. control; ${ }^{*} P<0.05,{ }^{* *} P<0.01$ vs. diabetes $(n=10)$. FBG fasting blood glucose, BUN blood urea nitrogen, $\mathrm{Cr}$ serum creatinine, $24-\mathrm{h}$ UP urine protein over $24 \mathrm{~h}$, PD polydatin.

renal function parameters in diabetic mice and the expression of Cx32 and Nox4 in the kidney. We observed that polydatin significantly reduced fasting blood glucose (FBG) levels, the kidney weight/body weight ratio, serum creatinine $(\mathrm{Cr})$ levels, blood urea nitrogen (BUN) levels and $24 \mathrm{~h}$ urinary protein (UP) levels in the diabetic mice and reversed the weight loss of diabetic mice (Fig. $6 a-f$ ). The results of PAS staining showed that polydatin attenuated glomerular hypertrophy and mesangial expansion in diabetic mice (Fig. $6 \mathrm{~g}$ ). Masson staining revealed that polydatin reduced the deposition of collagen fibers in the glomeruli of diabetic mice (Fig. 6h). In addition, polydatin increased the protein levels of $\mathrm{C} \times 32$ but reduced the protein levels of Nox4 and the extracellular matrix protein FN in the glomeruli of diabetic mice, as shown by immunohistochemical staining (Fig. 6i). Similarly, we observed that polydatin restored the expression of $\mathrm{C} \times 32$ and downregulated the expression of Nox4 in the kidneys of diabetic mice, as shown by Western blot analysis (Fig. 6j).
Polydatin reduces the expression of fibrotic factors in the kidneys of diabetic mice by inhibiting renal oxidative stress

There were significant decreases in the expression of $\mathrm{FN}$ and ICAM-1 (Fig. 7a, b), as well as additional fibrotic factors, including plasminogen activator inhibitor type 1 (PAl-1), connective tissue growth factor (CTGF), collagen IV (Col-IV) and TGF- $\beta$, in the kidneys of diabetic mice treated with polydatin (Fig. $7 c-f$ ). To further validate the effects of polydatin on renal oxidative stress, we detected the changes in two oxidative stress markers, namely, total SOD activity and MDA levels [36-38]. As shown in Fig. 7c-f, total SOD activity was reduced, and the levels of MDA were increased in the kidneys and serum of diabetic mice compared to those of control mice, and this effect was reversed by polydatin treatment. These data suggest that polydatin promotes K48-linked polyubiquitination and degradation of Nox4 to reduce renal oxidative stress levels by increasing the expression of Connexin32, thereby improving renal fibrosis in diabetic mice (Fig. 8). 
a
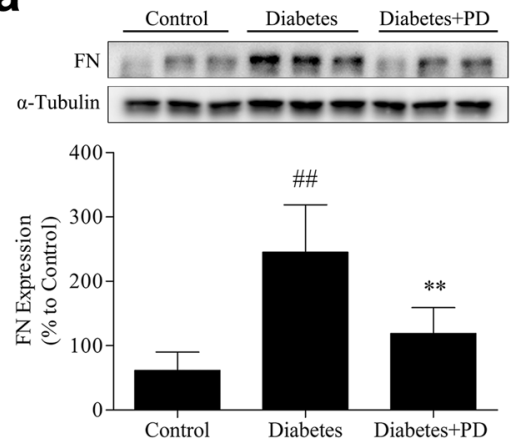

d
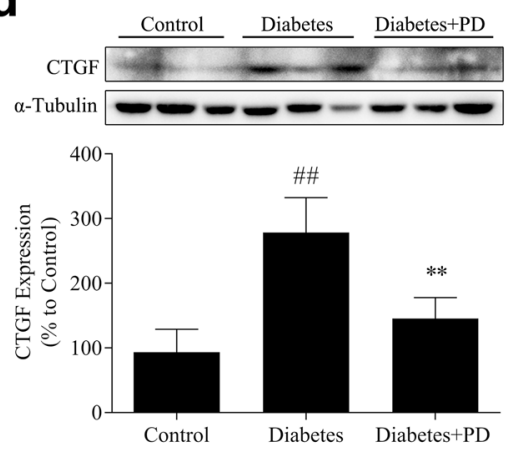

g

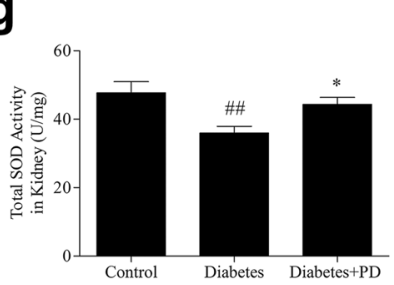

h b
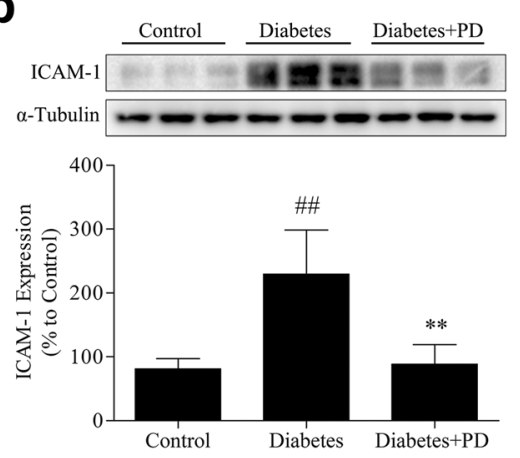

e
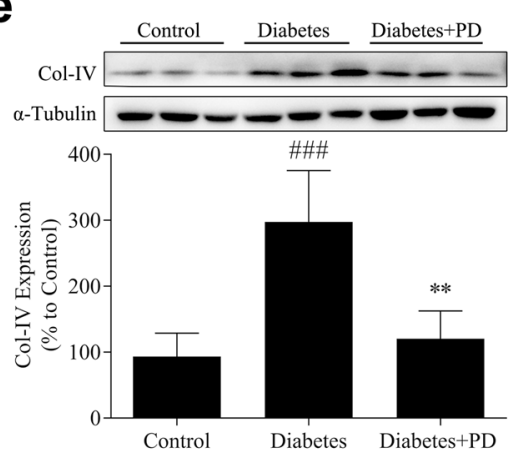

i
C
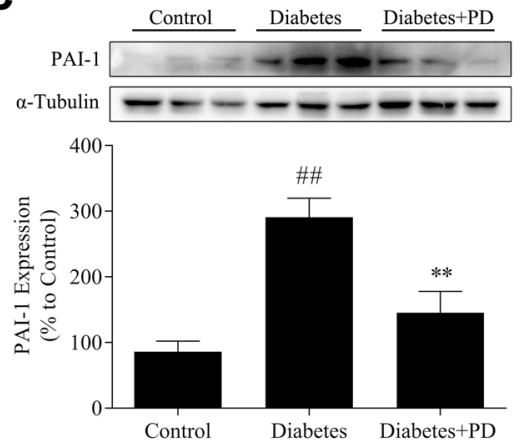

f
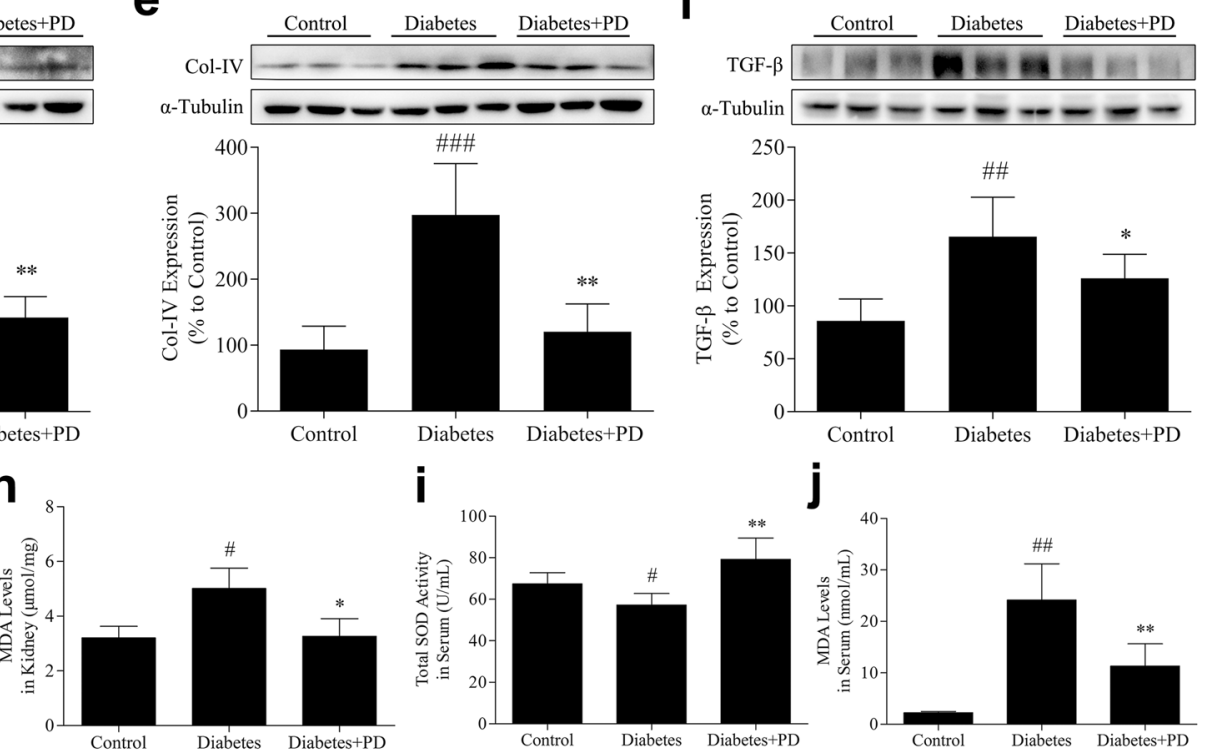

j

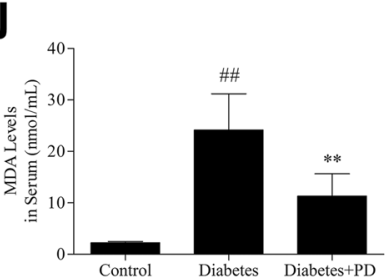

Fig. 7 Polydatin reduces the expression of fibrotic factors in the kidneys of diabetic mice, inhibiting renal oxidative stress. $a$, $b$ The expression levels of FN and ICAM-1 in the kidneys of mice were detected using Western blot analysis. c-f The effects of polydatin on the expression of additional fibrotic factors (PAI-1, CTGF, Col-IV and TGF- $\beta$ ). $\mathbf{g}-\mathbf{j}$ Polydatin treatment increased total SOD activity and reduced the levels of MDA in the kidneys and serum of diabetic mice; ${ }^{\#} P<0.05,{ }^{\# \#} P<0.01$, ${ }^{\# \#} P<0.001$ vs. control; ${ }^{*} P<0.05$, ${ }^{* *} P<0.01$ vs. diabetes $n=10$ ). PD polydatin.

\section{DISCUSSION}

Our previous studies have shown that polydatin can improve diabetic renal fibrosis by inhibiting oxidative stress [22, 23]. Nevertheless, the exact role of polydatin in diabetic renal fibrosis and the specific molecular mechanism of its regulatory effects on oxidative stress remain to be elucidated. Here, we aimed to investigate whether polydatin exerts antioxidative stress effects and protects against kidney injury by targeting the Cx32-Nox4 signaling pathway.

GMCs, intrinsic cells of the kidney, are critical in maintaining kidney structure and physiological functions [26, 27]. Hyperglycemia-induced ROS overproduction by upregulation of Nox4 contributes to inflammatory and fibrotic processes in the kidneys $[7,8]$. In this study, we confirmed that polydatin suppressed the expression of FN and ICAM-1 by reducing the production of ROS in HG-exposed GMCs, thereby attenuating diabetic renal fibrosis.

It is believed that the protein levels of Nox4 are mainly associated with posttranslational modifications, namely, K48linked ubiquitination, while the mRNA levels of Nox4 remain almost unchanged $[10,39]$. Nox4 deficiency ameliorates kidneys injury by reducing renal ROS production [40]. Here, we observed an increase in Nox4 expression in HG-exposed GMCs. Polydatin inhibited the expression of FN and ICAM-1 by reducing Nox4 expression. In addition, polydatin increased K48-linked polyubiquitination of Nox4. These findings suggest that polydatin may reduce the protein level of Nox4 by promoting its K48-linked polyubiquitination and degradation in the proteasome.

Recently, studies have shown that $\mathrm{Cx} 32$ has an inhibitory effect on oxidative stress during liver injury $[13,14]$. In addition, Cx32 reduces the expression and secretion of renin [16], which regulates the expression of TGF- $\beta 1$ in GMCs, thereby aggravating DN lesions [17]. Furthermore, we recently demonstrated that $\mathrm{C} \times 32$ ameliorates renal fibrosis in diabetic mice by promoting K48linked Nox4 polyubiquitination and degradation [18]. In addition, polydatin reduced the protein levels of FN and ICAM-1 in HGexposed GMCs by increasing the expression of Cx32. Furthermore, we observed that $\mathrm{C} \times 32$ was colocalized and interacted with Nox4 in GMCs and decreased the expression of Nox4. Importantly, Cx32 mediated the regulation of Nox4 K48-linked polyubiquitination by polydatin.

Moreover, in vivo experiments showed that Cx32 expression was downregulated in the kidneys of diabetic mice, while Nox4 expression was upregulated, and that these changes were accompanied by a decrease in total SOD activity and an increase in MDA levels in the kidneys and serum, which were reversed by polydatin treatment. Ultimately, polydatin reduced the protein levels of fibrotic factors in the kidneys and improved renal dysfunction and fibrosis in diabetic mice.

These results indicate that polydatin increases Cx32 expression, reduces Nox4 expression by promoting its K48-linked polyubiquitination, and decreases renal oxidative stress as well as fibrotic 


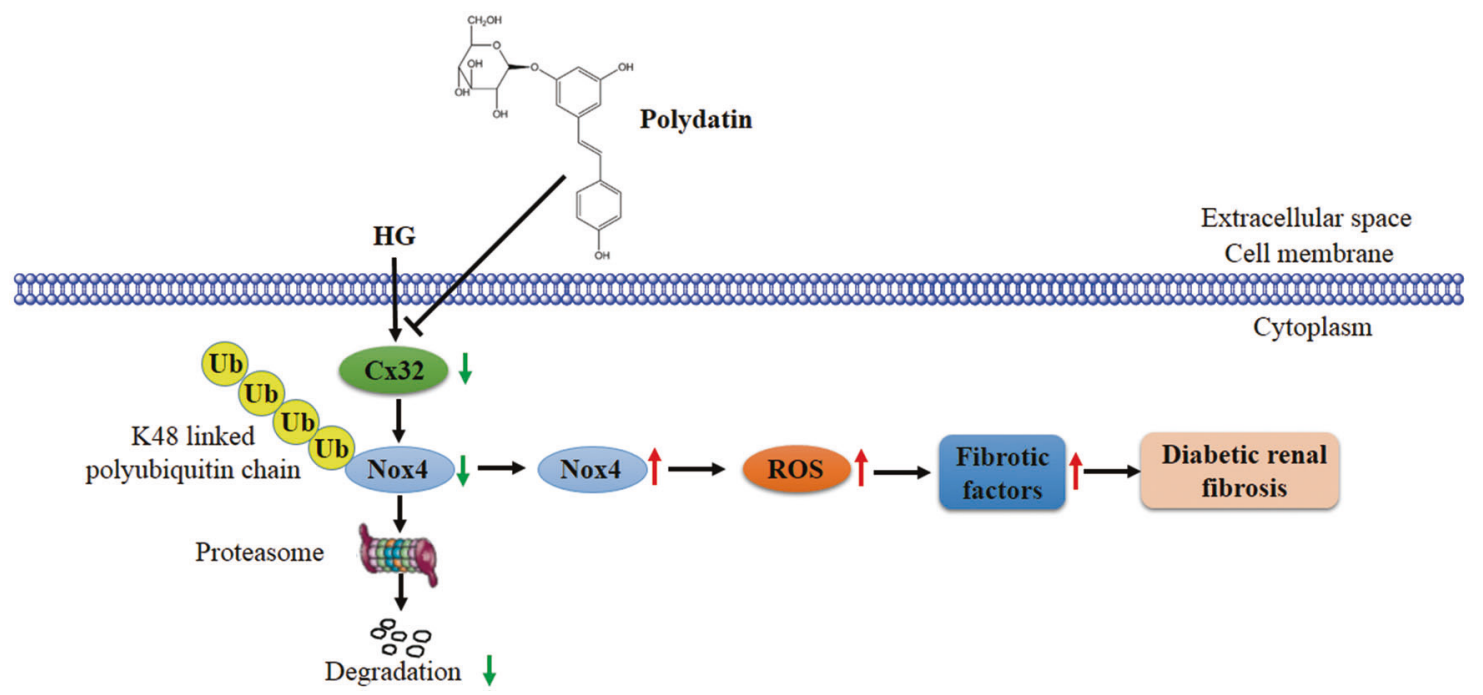

Fig. 8 Polydatin promotes K48-linked polyubiquitination and degradation of Nox4 to reduce renal oxidative stress levels by increasing the expression of Connexin32, thereby improving renal fibrosis in diabetic mice. Under normal conditions, Nox4 was mainly modified by K48-linked polyubiquitination and subsequently degraded by the proteasome. In diabetes or under high-glucose conditions, the protein expression of Cx32 was decreased, leading to the upregulation of Nox4 expression and resulting in intracellular ROS overproduction. Increased oxidative stress further promoted fibrotic factor expression, eventually exacerbating diabetic renal fibrosis, which was reversed by polydatin.

factor expression in HG-cultured GMCs and diabetic kidneys, ultimately ameliorating diabetic renal fibrosis.

\section{CONCLUSION}

Taken together, the in vitro and in vivo experiments suggested that polydatin promotes K48-linked polyubiquitination and degradation of Nox4 by restoring Cx32 expression, thereby decreasing renal oxidative stress levels and ultimately ameliorating the pathological progress of diabetic renal fibrosis. We further found that the mechanism by which polydatin reduces renal oxidative stress levels and attenuates diabetic renal fibrosis is closely related to regulation of the Cx32-Nox4 signaling pathway, providing some new experimental evidence for the potential clinical application of polydatin as a drug for DN.

\section{ACKNOWLEDGEMENTS}

This work was supported by research grants from the National Natural Science Foundation of China (grant number 81770816 and 81973375), the Key Project of Natural Science Foundation of Guangdong Province, China (grant number 2017A030311036), and the Youth Science Foundation of Guangxi Medical University, China (grant number GXMUYSF201903).

\section{AUTHOR CONTRIBUTIONS}

ZQC conceived and designed the study, performed the experiments, collected the data, analyzed and interpreted the data, and drafted the manuscript. XHS and XJL contributed to data collection and some of the experiments and drafted of the manuscript. ZCX contributed to language polishing. YY and ZYL contributed to the analysis and interpretation of the data. $\mathrm{HMX}$ and $\mathrm{MZ}$ contributed to the revision of the manuscript. SJQ and $\mathrm{HQH}$ contributed to the research design and supervision of the project.

\section{ADDITIONAL INFORMATION}

Competing interests: The authors declare no competing interests.

\section{REFERENCES}

1. Kanwar YS, Sun L, Xie P, Liu FY, Chen S. A glimpse of various pathogenetic mechanisms of diabetic nephropathy. Annu Rev Pathol. 2011;6:395-423.
2. Kim Y, Park CW. New therapeutic agents in diabetic nephropathy. Korean J Intern Med. 2017;32:11-25.

3. Alsaad KO, Herzenberg AM. Distinguishing diabetic nephropathy from other causes of glomerulosclerosis: an update. J Clin Pathol. 2007;60:18-26.

4. Jha JC, Banal C, Chow BS, Cooper ME, Jandeleit-Dahm K. Diabetes and kidney disease: role of oxidative stress. Antioxid Redox Signal. 2016;25:657-84.

5. Yang Q, Wu FR, Wang JN, Gao L, Jiang L, Li HD, et al. Nox4 in renal diseases: an update. Free Radic Biol Med. 2018;124:466-72.

6. Gorin Y, Block K. Nox4 and diabetic nephropathy: with a friend like this, who needs enemies? Free Radic Biol Med. 2013;61:130-42.

7. Jha JC, Gray SP, Barit D, Okabe J, El-Osta A, Namikoshi T, et al. Genetic targeting or pharmacologic inhibition of NADPH oxidase nox4 provides renoprotection in long-term diabetic nephropathy. J Am Soc Nephrol. 2014;25:1237-54.

8. Rhee EP. NADPH oxidase 4 at the nexus of diabetes, reactive oxygen species, and renal metabolism. J Am Soc Nephrol. 2016;27:337-9.

9. Palumbo S, Shin YJ, Ahmad K, Desai AA, Quijada H, Mohamed M, et al. Dysregulated Nox4 ubiquitination contributes to redox imbalance and age-related severity of acute lung injury. Am J Physiol Lung Cell Mol Physiol. 2017;312:L297-308.

10. Tsubouchi K, Araya J, Minagawa S, Hara H, Ichikawa A, Saito N, et al. Azithromycin attenuates myofibroblast differentiation and lung fibrosis development through proteasomal degradation of NOX4. Autophagy. 2017;13:1420-34.

11. Oyamada M, Takebe K, Oyamada $Y$. Regulation of connexin expression by transcription factors and epigenetic mechanisms. Biochim Biophys Acta. 2013;1828:118-33.

12. King TJ, Lampe PD. Mice deficient for the gap junction protein Connexin 32 exhibit increased radiation-induced tumorigenesis associated with elevated mitogen-activated protein kinase (p44/Erk1, p42/Erk2) activation. Carcinogenesis. 2004;25:669-80.

13. Cogliati B, Crespo Yanguas S, Da Silva TC, Aloia TP, Nogueira MS, Real-Lima MA, et al. Connexin32 deficiency exacerbates carbon tetrachloride-induced hepatocellular injury and liver fibrosis in mice. Toxicol Mech Methods. 2016;26:362-70.

14. Tiburcio TC, Willebrords J, da Silva TC, Pereira IV, Nogueira MS, Crespo Yanguas S, et al. Connexin32 deficiency is associated with liver injury, inflammation and oxidative stress in experimental non-alcoholic steatohepatitis. Clin Exp Pharmacol Physiol. 2017;44:197-206.

15. Pitre DA, Seifert JL, Bauer JA. Perineurium inflammation and altered connexin isoform expression in a rat model of diabetes related peripheral neuropathy. Neurosci Lett. 2001;303:67-71.

16. Arora MK, Singh UK. Molecular mechanisms in the pathogenesis of diabetic nephropathy: an update. Vasc Pharmacol. 2013;58:259-71.

17. Haefliger JA, Krattinger N, Martin D, Pedrazzini T, Capponi A, Doring B, et al. Connexin43-dependent mechanism modulates renin secretion and hypertension. J Clin Invest. 2006;116:405-13.

18. Chen Z, Sun X, Chen Q, Lan T, Huang K, Xiao H, et al. Connexin32 ameliorates renal fibrosis in diabetic mice by promoting K48-linked NADPH oxidase 4 polyubiquitination and degradation. Br J Pharmacol. 2020;177:145-60. 
19. Ravagnan G, De Filippis A, Carteni M, De Maria S, Cozza V, Petrazzuolo M, et al. Polydatin, a natural precursor of resveratrol, induces beta-defensin production and reduces inflammatory response. Inflammation. 2013;36:26-34.

20. Du QH, Peng C, Zhang H. Polydatin: a review of pharmacology and pharmacokinetics. Pharm Biol. 2013;51:1347-54.

21. Hao J, Chen C, Huang K, Huang J, Li J, Liu P, et al. Polydatin improves glucose and lipid metabolism in experimental diabetes through activating the Akt signaling pathway. Eur J Pharmacol. 2014;745:152-65.

22. Gong W, Li J, Chen Z, Huang J, Chen Q, Cai W, et al. Polydatin promotes Nrf2-ARE anti-oxidative pathway through activating CKIP-1 to resist HG-induced up-regulation of FN and ICAM-1 in GMCs and diabetic mice kidneys. Free Radic Biol Med. 2017;106:393-405.

23. Huang $\mathrm{K}$, Chen C, Hao J, Huang J, Wang S, Liu P, et al. Polydatin promotes Nrf2-ARE anti-oxidative pathway through activating Sirt1 to resist AGEs-induced upregulation of fibronetin and transforming growth factor-beta1 in rat glomerular messangial cells. Mol Cell Endocrinol. 2015;399:178-89.

24. Chen Z, Xie X, Huang J, Gong W, Zhu X, Chen Q, et al. Connexin43 regulates high glucose-induced expression of fibronectin, ICAM-1 and TGF-beta1 via Nrf2/ARE pathway in glomerular mesangial cells. Free Radic Biol Med. 2017;102: 77-86.

25. Jiang Q, Liu P, Wu X, Liu W, Shen X, Lan T, et al. Berberine attenuates lipopolysaccharide-induced extracelluar matrix accumulation and inflammation in rat mesangial cells: involvement of NF-kappaB signaling pathway. Mol Cell Endocrinol. 2011;331:34-40.

26. Abboud HE. Mesangial cell biology. Exp Cell Res. 2012;318:979-85.

27. Schlondorff D, Banas B. The mesangial cell revisited: no cell is an island. J Am Soc Nephrol. 2009;20:1179-87.

28. Cove-Smith A, Hendry BM. The regulation of mesangial cell proliferation. Nephron Exp Nephrol. 2008;108:e74-9.

29. Lai Y, Zhou C, Huang P, Dong Z, Mo C, Xie L, et al. Polydatin alleviated alcoholic liver injury in zebrafish larvae through ameliorating lipid metabolism and oxidative stress. J Pharmacol Sci. 2018;138:46-53.

30. Lv T, Shen L, Yang L, Diao W, Yang Z, Zhang Y, et al. Polydatin ameliorates dextran sulfate sodium-induced colitis by decreasing oxidative stress and apoptosis partially via Sonic hedgehog signaling pathway. Int Immunopharmacol. 2018;64:256-63.

31. Lv R, Du L, Zhang L, Zhang Z. Polydatin attenuates spinal cord injury in rats by inhibiting oxidative stress and microglia apoptosis via Nrf2/HO-1 pathway. Life Sci. 2019;217:119-27.

32. Jha JC, Banal C, Okabe J, Gray SP, Hettige T, Chow BSM, et al. NADPH oxidase Nox5 accelerates renal injury in diabetic nephropathy. Diabetes. 2017;66:2691-703.

33. Ni Z, Tao L, Xiaohui X, Zelin Z, Jiangang L, Zhao S, et al. Polydatin impairs mitochondria fitness and ameliorates podocyte injury by suppressing Drp1 expression. J Cell Physiol. 2017;232:2776-87.

34. Sedeek M, Callera G, Montezano A, Gutsol A, Heitz F, Szyndralewiez C, et al. Critical role of Nox4-based NADPH oxidase in glucose-induced oxidative stress in the kidney: implications in type 2 diabetic nephropathy. Am J Physiol Renal Physiol. 2010;299:F1348-58.

35. Martyn KD, Frederick LM, von Loehneysen K, Dinauer MC, Knaus UG. Functional analysis of Nox4 reveals unique characteristics compared to other NADPH oxidases. Cell Signal. 2006;18:69-82.

36. Amin MM, Rafiei N, Poursafa P, Ebrahimpour K, Mozafarian N, Shoshtari-Yeganeh $B$, et al. Association of benzene exposure with insulin resistance, SOD, and MDA as markers of oxidative stress in children and adolescents. Environ Sci Pollut Res Int. 2018;25:34046-52.

37. Hajiluian G, Abbasalizad Farhangi M, Nameni G, Shahabi P, Megari-Abbasi M. Oxidative stress-induced cognitive impairment in obesity can be reversed by vitamin D administration in rats. Nutr Neurosci. 2018;21:744-52.

38. Wang H, Gao N, Li Z, Yang Z, Zhang T. Autophagy alleviates melamine-induced cell death in PC12 cells via decreasing ROS level. Mol Neurobiol. 2016;53:1718-29.

39. New DD, Block K, Bhandhari B, Gorin Y, Abboud HE. IGF-I increases the expression of fibronectin by Nox4-dependent Akt phosphorylation in renal tubular epithelial cells. Am J Physiol Cell Physiol. 2012;302:C122-30.

40. Thallas-Bonke V, Jha JC, Gray SP, Barit D, Haller $H$, Schmidt HH, et al. Nox-4 deletion reduces oxidative stress and injury by PKC-alpha-associated mechanisms in diabetic nephropathy. Physiol Rep. 2014;2:e12192. https://doi.org/ 10.14814 /phy2.12192. 